\title{
Nonlinear Dynamics Model of Crowd Panic Propagation under Disaster Conditions
}

\author{
Rongyong Zhao ${ }^{1}$, Daheng Dong, ${ }^{1, *}$, Qianshan $\mathrm{Hu}^{1}$, Cuiling $\mathrm{Li}^{2}$ and Dong Wang ${ }^{1}$ \\ ${ }^{1}$ CIMS Research Center, Tongji University, Shanghai 201804, China \\ ${ }^{2}$ College of Electronic and Information Engineering, Tongji University, Shanghai 201804, China \\ ${ }^{*}$ Corresponding author
}

\begin{abstract}
In disaster conditions, people often fall in panic. When the population panics raise to a certain extent, they can aggravate the confusion degree of crowd evacuation, leading to greater casualties further. Therefore, this paper proposes a nonlinear dynamic model describing crowd panic propagation, to support theoretically the effective evacuation guidance and control of panic-stricken crowd.

Based on the natural similarity between the panic transmission and the ball collision, this paper introduces the ball collision model to describe the panic transmission characteristics based on the traditional dynamics model and transforms the evacuation panic transmission problem into a linear dynamic ball collision model. The degree of panic changes the force between evacuee individuals and determines the expansion degree of the ball volume, then changes the diameter of the ball. The amount of change in momentum measures the degree of panic.
\end{abstract}

Using the non-linear dynamics model of panic propagation in this paper, we can systematically analyze the impact of panic transmission on population evacuation by adjusting various elements of evacuation scenarios.

Keywords-nonlinear dynamic model; control; disaster condition; panic; dynamic ball model

\section{INTRODUCTION}

Disasters can lead to swarm panic during the evacuation crowd, which is not only the cause of stampede but also the cause leading to the expansion of the consequences. In recent years, scholars pay more and more attention to the multidisciplinary research, such as comprehensive social science, management science, information science and system science [1]. Further research has been conducted to explore the formation and propagation of panic in evacuation and panic's effect on stability of the crowd. Therefore, simulation results have been becoming closer to the real situation. However, panic psychology is complex, with the character of timedegeneration and non-replicability. What's more, evacuation of crowd is a complex process of non-linear, unstructured and self-organizing [2]. Therefore, it is difficult to analyze the instability process and evolution mechanism with the classical Lyapunov function [3-5].

Domestic research on panic has entered its infancy [6-8], but there are some shortcomings. On the one hand, there are few microanalysis, and the existing models mostly study the problem of panic propagation from a macro perspective. On the other hand, the classical model of panic propagation lacks timevariability and has not yet formed a method of operational analysis. What's more, the classic model which reflects the personal panic degree is fixed. In reality, the degree of panic in the crowd is "situational dependence". In the situation without guidance, the individual's panic degree increases with the severity of the disaster. In the situation with guidance, the guiding behavior has a stabilizing effect on the panic and the individual's panic will be alleviated. Due to these features above, panic needs dynamics analysis.

\section{TWo DIMENSIONAL PANIC PROPAgATION MODEL}

\section{A. Under Normal Circumstances}

The normal circumstance refers to the natural movement state of the people without disasters. The distance between evacuation individuals is randomly distributed, and there exists social force (self-thrust and thrust) between evacuation individuals. Based on the theories mentioned above, this paper establishes a classic social force model. This method transforms the social force model into momentum to analyze the panic propagation. As shown in Eq. (1).

$$
\vec{P}_{1}=\vec{P}_{2}
$$

where $\vec{P}_{1}$ is the total momentum before the collision, and $\vec{P}_{2}$ is the total momentum after the collision. $m_{i}$ is the mass of the $i$ th evacuation individual, and $\vec{v}_{i}$ is the actual speed of the $i$ th evacuation individual.

\section{B. Panic Propagation Model}

When disasters occur, a large number of people usually accumulates in a region, and then the individuals want to reach a certain destination $\vec{l}_{i}^{0}$ as much as possible [9]. Therefore, the evacuation individuals will not detour. That is to say, the individual can walk along the shortest path as far as possible. This road is usually a polygon shape, and it's edges satisfy $\vec{l}_{i}{ }^{1}, \vec{l}_{i}{ }^{2} \ldots \vec{l}_{i}{ }^{n}=\vec{l}_{i}{ }^{0}$. If $\vec{l}_{i}{ }^{k}$ is the next edge of the polygon connection, then the evacuation individual's desired direction $\vec{e}_{i}(t)$ can be represented by Eq. (2). 


$$
\vec{e}_{i}(t)=\frac{\vec{l}_{i}^{k}-\vec{l}_{i}(t)}{\left|\vec{l}_{i}^{k}-\vec{l}_{i}(t)\right|}
$$

where $\vec{l}_{i}(t)$ represents the actual location of the evacuation individual $i$ at the moment $t$. Accurately speaking, the target of the evacuation individual is usually a door or a region, not a specific point $\vec{l}_{i}^{k}$. In this case, the evacuation individuals should guide themselves to a door or the nearest point $\vec{l}_{i}^{k}$ related to a certain area at any moment.

If the movement of an evacuation individual is not disturbed, they will move in its expected speed $v_{i}^{0}$ along the desired direction $\vec{e}_{i}(t)$. Due to interference, the necessary deceleration or acceleration process is made, which makes the actual speed $\vec{v}_{i}^{0}(t)=v_{i}^{0} \vec{e}_{i}(t)$ of the evacuation individuals deviate from the expected speed, which leads to the trend that the evacuation individuals' speed are approaching $\vec{v}_{i}^{0}(t)$ in a particular relaxation time of $\tau_{i}$. It can be expressed by Eq. (3).

$$
\vec{f}_{i}^{0}\left(\vec{v}_{i}, v_{i}^{0} \vec{e}_{i}\right)=\frac{1}{\tau_{i}}\left(v_{i}^{0} \vec{e}_{i}-\vec{v}_{i}\right)
$$

where $\vec{f}_{i}^{0}\left(\vec{v}_{i}, v_{i}^{0} \vec{e}_{i}\right)$ is self-thrust, which is not generated from external factors, but from each individual itself.

Out-thrust is the force of interaction deriving from collide with each other and the boundaries? The movement of evacuation individuals is influenced by another evacuation individual, especially evacuation individuals keep a certain distance from other evacuation individuals [10], which depends on the evacuation individuals' desired speed $v_{i}^{0}$. Generally speaking, the closer an individual is to a stranger, the more uncomfortable they feel. Thus, the exclusion of evacuees from $j$ to $i$ is occurred. As shown in Eq. (4).

$$
\vec{f}_{i j}\left(\vec{l}_{i j}\right)=-\Delta_{\vec{l}_{i j}} V_{i j}\left[b\left(\vec{l}_{i j}\right)\right]
$$

where the exclusion potential $V_{i j}(b)$ is a monotonically increasing function of $b$, this repulsion potential has the equipotential lines in elliptic form, pointing to the direction of motion. Evacuation Individuals prepare a space for the next step in walking, which is also considered by other evacuation individuals, where $b$ denotes the semi-minor axis of the ellipse and b Satisfies Eq. (5).

$$
2 b=\sqrt{\left(\left|\vec{l}_{i j}\right|+\left|\vec{l}_{i j}-\vec{v}_{j} \Delta t \vec{e}_{j}\right|\right)^{2}-\left(\vec{v}_{j} \Delta t\right)^{2}}
$$

where $\vec{l}_{i j}=\vec{l}_{i}-\vec{l}_{j}$.

Sometimes, evacuated individuals are attracted to other people (such as family, friends, etc.) or objects (such as windows). The attraction of these locations $\vec{r}_{k}$ can be expressed by the monotonically increasing potential $W_{i k}\left(\left|\vec{l}_{i k}\right|, t\right)$ of attraction in a similar way as the repulsion potential. As shown in Eq. (6).

$$
\vec{f}_{i k}\left(\left|\vec{l}_{i k}\right|, t\right)=-\Delta_{\vec{l}_{i k}} W_{i k}\left(\left|\vec{l}_{i k}\right|, t\right) \quad\left(\vec{l}_{i k}=\vec{l}_{i}-\vec{l}_{k}\right)
$$

The main difference is that the attractiveness $\left|\vec{f}_{i k}\right|$ normally decreases over time. Therefore, the mutual attraction is the reason for the form of evacuation individual groups [11].

However, the Eq. above for attracting and repulsion is only used in a state that is perceived in the desired direction $\vec{e}_{i}(t)$ of the movement. The situation behind the evacuating individual will be fainted, and we denote by c $(0<\mathrm{c}<1)$ [12]. To account for this sensitive role, introduce a measure that depends on direction. As shown in Eq. (7).

$$
W(\vec{e}, \vec{f})=\left\{\begin{array}{lc}
1 & \vec{e} \cdot \vec{f} \geq|\vec{f}| \cos \varphi \\
c & \text { other }
\end{array}\right.
$$

In summary, the exclusion or attraction of evacuees is given by Eq. (8) and (9).

$$
\begin{array}{r}
\vec{f}_{i j}\left(\vec{e}_{i}, \vec{l}_{i}-\vec{l}_{j}\right)=w\left(\vec{e}_{i},-\vec{f}_{i j}\right) \vec{f}_{i j}\left(\vec{l}_{i}-\vec{l}_{j}\right) \\
\vec{f}_{i k}\left(\vec{e}_{i}, \vec{l}_{i}-\vec{l}_{k}, t\right)=w\left(\vec{e}_{i}, \vec{f}_{i k}\right) \vec{f}_{i k}\left(\vec{l}_{i}-\vec{l}_{k}, t\right)
\end{array}
$$

Since evacuation individuals decide where they want to move, this paper establishes the equation for the total motivation $\vec{f}_{i j}(t)$ evacuated by individuals. As shown in Eq. (10).

$$
\vec{f}_{i j}(t)=\vec{f}_{i}^{0}\left(\vec{V}_{i}, v_{i}^{0} \vec{e}_{i}\right)+\sum_{j} \vec{f}_{i j}\left(\vec{e}_{i}, \vec{I}_{i}-\vec{I}_{j}\right)+\sum_{k} \vec{f}_{i k}\left(\vec{e}_{i}, \vec{I}_{i}-\vec{I}_{k}, t\right)
$$

Evacuating individuals also maintain a certain distance from the boundaries, walls, streets, obstacles of the building. The closer evacuating individuals get to the obstacle, the more disastrous they are. That is to say, the evacuee will have to pay more attention to the danger of being injured [13]. Therefore, a boundary W elicits a repulsive effect, as shown in Eq. (11). 


$$
\vec{f}_{i w}=-\Delta_{\bar{l}_{i w}} U_{i w}\left(\left|\vec{l}_{i w}\right|\right)
$$

where $\vec{l}_{i w}=\vec{l}_{i}-\vec{l}_{w}^{i}$. There is an exclusive and monotonically increasing potential $U_{i w}\left(\vec{l}_{i w}\right)$, and $\vec{r}_{w}^{i}$ represents the closest location between the wall $W$ and the evacuation individual $i$.

According to Helbing's 'Psychological-Behavioral' panic fluctuation model [3], the degree of panic was calculated from the changes of stress level and speed of evacuees, and the panic degree was mapped to the diameter of the ball. The level of panic can be expressed as Eq. (12).

$$
m_{i} \frac{d \vec{v}_{i}}{d t}=m_{i} \frac{v_{i}^{0}(t) \vec{e}_{i}^{0}(t)-\vec{v}_{i}(t)}{\tau_{i}}+\vec{f}_{i j}(t)
$$

where $m_{i}$ is the mass of the $i$ th evacuation individual, $v_{i}^{0}$ is the ideal speed, $\vec{e}_{i}^{0}$ is the set direction, $\vec{v}_{i}$ is the actual speed and $\tau_{i}$ is the characteristic time

$$
\vec{f}_{i j}=\left\{A_{i} \exp \left[\left(d_{i j}-d_{c i j}\right) / B_{i}\right]+k g\left(d_{i j}-d_{c i j}\right)\right\} \vec{n}_{i j}+\gamma g\left(d_{i j}-d_{c i j}\right) \Delta v_{j i}^{t} \vec{t}_{i j}
$$

$$
\left.\bar{f}_{i w}=\left\{A_{i} \exp \left[\left(r_{i}-d_{i w}\right) / B_{i}\right]+k g\left(r_{i}-d_{i w}\right)\right\} \bar{n}_{i w}-\gamma g\left(r_{i}-d_{i v}\right)\left(v_{i} \cdot \vec{t}_{i w}\right)\right)_{i w}
$$

where $A_{i} 、 B_{i}$ are constants, $d_{c i j}$ is the center distance of the evacuation individuals' mass, $d_{i j}=\left\|\vec{r}_{i}-\vec{r}_{j}\right\|$ is the distance between two evacuation individuals, $\vec{n}_{i j}=\left(n_{i j}^{1}, n_{i j}^{2}\right)=\left(\vec{r}_{i}-\vec{r}_{j}\right) / d_{c i j}$ is the standard vector from $j$ to $i$. If $d_{c i j}<d_{i j}$, collisions between evacuation individuals will occur[14]. $\vec{t}_{i j}$ refers to the tangential direction, and $\Delta v_{j i}^{t}=\left(\vec{v}_{j}-\vec{v}_{i}\right) \cdot \vec{t}_{i j}$ is the variation of the tangential velocity. $k\left(d_{i j}-d_{c i j}\right) \vec{n}_{i j}$ is mass force, $\gamma\left(d_{i j}-d_{c i j}\right) \Delta v_{j i}^{t} \vec{t}_{i j}$ is sliding friction force. $d_{i w}$ is the distance between the $i$ th evacuation individual and the wall, $\vec{n}_{i w}$ is the vertical direction, and $\vec{t}_{i w}$ is the tangential direction[15]. $\vec{t}_{i j}$ is the tangential direction of $\vec{n}_{i j}$, and $\vec{n}_{i j}$ is the standard vector from evacuation individual $j$ to the $i . r_{i}$ is the diameter of the $i$ th evacuated individual. $g(x)$ is a function. If the pedestrian collides, $g(x)=0$; otherwise $g(x)=x$.

$$
\Delta D_{i}=2\left(d_{c i j}-d_{i j}-\left|\vec{r}_{j}\right|-\left|\vec{r}_{i}\right|\right)
$$

where $\Delta D_{i}$ is the amount of change in ball's diameter in a case of panic[17].

In a panic situation, the ball diameter changes, resulting in changes in the mass of evacuation individuals. As shown in Eq. (16).

$$
\Delta m_{i}=\rho_{i}\left\{\frac{4}{3} \pi\left(\left(D_{i}+\Delta D_{i}\right) / 2\right)^{3}-\frac{4}{3} \pi\left(D_{i} / 2\right)^{3}\right\}
$$

where $\Delta m_{i}$ is the amount of change in the mass of the ball, and $\rho_{i}$ is the density of the ball[18]. $\Delta \vec{P}_{i}$ can be expressed by (17).

$$
\Delta \vec{P}_{i}=\Delta m_{i} \vec{V}_{i}
$$

$\Delta \vec{P}_{i x}$ and $\Delta \vec{P}_{i y}$ are respectively the projection of the momentum variation on $X$-axis and the $y$-axis. The vector addition is performed on the $X$-axis and the $Y$-axis to obtain the value of $\Delta \vec{P}_{i}$.

$$
\Delta \vec{P}_{i x}=\Delta m_{i} \vec{v}_{i x}
$$

$$
\Delta \vec{P}_{i y}=\Delta m_{i} \vec{v}_{i y}
$$

where $\vec{v}_{i x}=\vec{v}_{i} \cos \alpha$ is the projection of velocity on the $\mathrm{x}$ axis, $\vec{v}_{i y}=\vec{v}_{i} \sin \alpha$ is the projection of velocity on the y-axis, $\Delta \vec{P}_{i x}$ is the projection of momentum on the x-axis, $\Delta \vec{P}_{i y}$ is the projection of momentum on the y-axis and $\alpha=\arctan \frac{\left|\vec{v}_{y}\right|}{\left|\vec{v}_{k}\right|}$ is the angle between vector $\vec{v}_{i}$ and x-axis. In this case, the total momentum $\vec{P}_{2}$ after the collision can be expressed by the Eq. (20).

$$
\vec{P}_{2}=\vec{P}_{1}+\sum \Delta \vec{P}_{i}
$$

\section{SimUlATION EXAMPLE}

There are three evacuation individuals, as shown in Figure I under normal circumstances, and panic situation is shown in Figure II . This research assume $m_{i}=80 \mathrm{~kg}$, $\vec{v}_{i}^{0}=0.8 \mathrm{~m} / \mathrm{s}$, be concluded that the momentum of has 
increased by 20 after the propagation of two person, accounting for $17.7 \%$ of the pre-panic momentum. That is to say, the panic degree of evacuation individual has increases by $17.7 \%$.

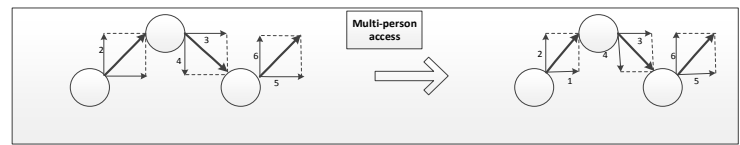

FIGURE I. TWO-DIMENSIONAL EVACUATION OF INDIVIDUAL MOTIONS WITHOUT PANIC

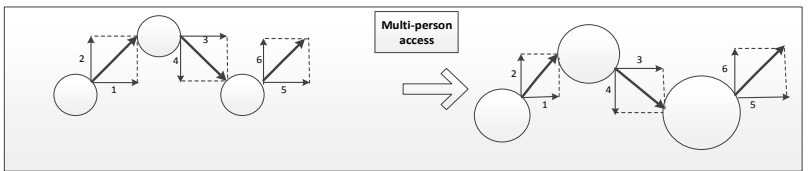

FIGURE II . EVACUATE INDIVIDUALS IN TWO-DIMENSIONAL PANIC CASE

\section{CONCLUSION}

In order to study the influence of panic psychology on evacuation of population effectively, this paper provides a new method nonlinear dynamics model for panic propagation based on ball collision principle, which provides method and support for emergency evacuation of panic population under disaster conditions. This nonlinear dynamics model of panic transmission has a very important role on crowd evacuation in the large-scale urban area [19].

\section{ACKNOWLEDGMENT}

This work is supported by the National Natural Science Foundation of China (Grant No. 71373178), Natural Science Foundation of Shanghai, China (Grant No. 13ZR1444700 and 15ZR1420100) and Model Course Construction Project of English Teaching Program for Foreign Students of Shanghai, China (Grant No. 2015-137). "Mechanism Analysis” Excellent experiment project of Tongji University (Grant No. 20160000025035340).

\section{REFERENCES}

[1] Manley, M. and Y.S. Kim, Modeling emergency evacuation of individuals with disabilities (exitus): An agent-based public decision support system. Expert Systems with Applications, 2012. 39(9):83008311.

[2] Luh, P.B., et al., Modeling and Optimization of Building Emergency Evacuation Considering Blocking Effects on Crowd Movement. Ieee Transactions on Automation Science and Engineering, 2012. 9(4): 687700.

[3] Helbing, D., L. Buzna, et al. Self-organized pedestrian crowd dynamics Experiments, simulations, and design solutions. Transportation Science, 2005,39(1):1-24

[4] Helbing, D., I. J. Farkas, et al. Simulation of pedestrian crowds in normal and evacuation situations. Pedestrian and Evacuation Dynamics, 2002: 21-58.

[5] Heliovaara, S., et al., Pedestrian behavior and exit selection in evacuation of a corridor - An experimental study. Safety Science, 2012. 50(2):221-227.

[6] WANG,Lin,Z. G.-Q., CHEN TIan-Lun. Self-Organized Criticality Analysis of Earthquake Model Based on Heterogeneous Networks. Commun. Theor.Phys.2011,55(1):89-94.
[7] Li, J.F., et al., Spatial evolution character of multi-objective evolutionary algorithm based on self-organized criticality theory. Physica a-Statistical Mechanics and Its Applications, 2012. 391(22): 490-5499.

[8] Giometto, A. and H.J. Jensen, Connecting the microdynamics to the emergent macrovariables: Self-organized criticality and absorbing phase transitions in the deterministic lattice gas. Physical Review E, 2012 85(1).

[9] D.Helbing,in Natual Structures.Principles,Strategies,and Models in Architecture and Nature, sonderforschungsbereich ,230, stuttgart, 1992, Pt, pp.93-98.

[10] A.E.Scheflen and N Ashcraft,Human Territories: How We Behave in Space-Time.Prentice-Hall,Englewood Cll-iffs,NJ,1976.

[11] R.D.Freimuth and L.Lam,in Modeling Complex Phenomena,edited by L.Lam and V.Naroditsky,Springer,New York,1992.

[12] D.R.Kayser, L.K.Aberle, R.D.Pochy, and L.Lam, Physica A 191,17,1992.

[13] H.J.Payne,in Mathematical Models of Public System,Simulation Council Proceeding,1971,vol.1,p.51.

[14] Lim, G.J., et al., A capacitated network flow optimization approach for short notice evacuation planning. European Journal of Operational Research, 2012. 223(1): 234-2a

[15] Qianshan Hu, Rongyong Zhao, Cuiling Li,Dong Wang, Xiangke Tian, Qin Zhang. Stability analysis for crowd evacuation based on selforganized criticality theory. Proceedings on Service System Engineering Conference \& Symposium on Analysis and Risk 2017, July 7th9th,2017,Shanhai,China.ISBN 978-1-926642-17-8.78-83.

[16] Dong Wang,Rongyong Zhao,Cuiling Li,Qianshan Hu, Xiangke Tian, Qin Zhang.Simulation and analysis of T-shaped road Junction stampede using macroscopic model. Proceedings on Service System Engineering Conference \& Symposium on Analysis and Risk 2017, July 7th9th,Shanhai,China,2017.ISBN 978-1-926642-17-8.71-77.

[17] Zhao, Rongyong; Wang, Jian; Ling, Weiqing.Crowd evacuation stability based on Self-organized Criticality Theory. Applied Mechanics and Materials.2014.Vol.2403.501-504.

[18] RongYong Zhao, Cuiling Li , Wei QingLing, YunLongMa ,A Rough Set based Knowledge Discovery Model for Crowd Evacuation a Fire Emergency, WIT Transactions on Information and Communication Technologies, 2014.47:92-96

[19] Rongyong ZHAO, Cuiling LI. Self-organized Criticality Theory and its Potential Application in High Density Pedestrian Evacuation Simulation in Public Buildings.2014. Applied Mechanics and Materials Vol. 501504, 2432-2435 